\title{
Engagement militant et phénomènes de radicalisation chez les Kurdes de Turquie
}

Militancy and radicalization among Turkish Kurds

\section{Gilles Dorronsoro et Olivier Grojean}

\section{(2) OpenEdition}

Journals

Édition électronique

URL : http://journals.openedition.org/ejts/198

DOI : $10.4000 /$ ejts. 198

ISSN : 1773-0546

Éditeur

EJTS

\section{Référence électronique}

Gilles Dorronsoro et Olivier Grojean, «Engagement militant et phénomènes de radicalisation chez les Kurdes de Turquie », European Journal of Turkish Studies [En ligne], Liste complète, mis en ligne le 04 août 2009, consulté le 19 février 2020. URL : http://journals.openedition.org/ejts/198; DOI : 10.4000/ ejts. 198 
Citation : Dorronsoro, Gilles ; Grojean, Olivier (2004) 'Engagement militant et phénomènes de radicalisation chez les Kurdes de Turquie', European Journal of Turkish Studies, URL: :

http://www.ejts.org/document198.html

To quote a passage, use paragraph (§).

\title{
Engagement militant et phénomènes de radicalisation chez les Kurdes de Turquie
}

Gilles Dorronsoro, Olivier Grojean

\begin{abstract}
Why and how do people engage in a radical movement? To answer this question, this paper analyses two Kurdish movements in Turkey, the PKK and the Hizbullah, under three different aspects. First, the constitution of radical groups is explained as a way for political entrepreneurs without social and economic capital to mobilize ressources. Radicalisation is also results of the competition with others groups belonging to the same political field. Secondly, personal dispositions are actualised during the process of adhesion. Here, the first group of militants linked by strong and multiple links has to be distinguished from the second generation, more likely to be made up by isolated individuals. Finally, the paper analyses two ways to bridge the old values of the primary socialization and the new world of the party: values such as charism and honor (namus) work in both universes and biographies (or autobiographies) allow individuals to recreate a coherence in their self-perception.
\end{abstract}




\section{Introduction ${ }^{1}$}

Comment les mobilisations en faveur d'un projet de contre-société émergentelles ? Si cette question est depuis longtemps le souci des avant-gardes révolutionnaires, elle reste finalement peu étudiée par la sociologie de l'engagement. Les notions de radicalité et de radicalisation peuvent s'avérer utiles lorsque l'on tente de comprendre l'adhésion d'une population à ce type de mouvement.

[2] La radicalité sera définie comme un ensemble de pratiques - dont les discours - qui instaurent une rupture entre une sodalité (un regroupement volontaire d'individus) et le reste de la société. Cette rupture est au principe de la constitution d'une organisation qui propose une alternative révolutionnaire à l'ordre social. L'univers de sens qui a cours au sein du mouvement est différent et, sur de nombreux points, opposé aux valeurs dominantes de la société. Au-delà de l'adhésion intellectuelle à une idéologie, la radicalisation peut alors permettre de décrire le processus qui conduit de l'univers de sens de départ à celui qui est institué au sein de l'organisation. La radicalisation d'un mouvement est de ce fait comprise comme une évolution visant à séparer le groupe de l'univers social dans lequel il s'inscrit par l'adoption et la mise en place volontaire d'un projet de contre-société. Elle est à différencier de la radicalisation individuelle qui suppose pour les adhérents une rupture avec l'ancien monde et une réforme profonde de leur habitus, sur le mode des conversions religieuses.

[3] En ce sens, la notion de radicalité est à comprendre comme un idéal-type, utilisable tant pour décrire des organisations que les militants qui les composent. Le degré de fermeture du groupe peut en effet varier: si de nombreuses d'organisations ont un discours radical, seules quelques-unes développent effectivement les structures d'une contre-société. La radicalisation des individus peut également s'effectuer par paliers, au moins quand l'organisation, devenue complexe, offre différents modes d'investissements, ce qui nuance l'idée d'une rupture toujours parfaitement nette entre les deux univers.

${ }^{1}$ Les auteurs remercient Benoit Fliche et Elise Massicard de leurs nombreux commentaires. 
Dorronsoro, Gilles ; Grojean, Olivier (2004) 'Engagement militant et phénomènes de radicalisation chez les Kurdes de Turquie', European Journal of Turkish Studies, URL: http://www.ejts.org/document198.html To quote a passage, use paragraph (§).

Enfin, et ce point apparaît fondamental, la radicalisation n'implique pas mécaniquement des modes d'action spécifiques comme, par exemple, la violence.

[4] Si de nombreux mouvement radicaux émergent dans les années 1960 et 1970 en Turquie, nous centrerons ici notre analyse uniquement sur les mobilisations kurdes. Les provinces majoritairement kurdes de l'est et du sud-est de la Turquie forment en effet un sous-espace politique progressivement autonomisé (Dorronsoro 1999) qui constitue un terrain propice à l'observation des phénomènes de radicalisation. Depuis les années 1960, le sud-est est le théâtre de transformations structurelles rapides. En moins de deux générations, une urbanisation et une modernisation accélérées ont bouleversé la société villageoise, restructuré l'ensemble des mécanismes de régulation sociale et favorisé la politisation rapide des générations montantes. Les années 1960 et 1970, marquées par une violence politique croissante dans l'ensemble de la Turquie, ont vu la formation de nombreux groupes d'étudiants et de lycéens kurdes marxistes, et plus rarement islamistes, sous forme d'associations culturelles tolérées ou de partis clandestins. La forte migration des Kurdes, notamment vers l'Allemagne, permet à certains groupes de s'implanter durablement en Europe. Contrairement au reste de la Turquie, où les groupes radicaux sont marginalisés dès le coup d'État de 1980, l'audience des groupes kurdes s'élargit à d'autres groupes sociaux au cours des années $1980^{3}$.

[5] Notre terrain sera donc multiple, à la fois par les partis considérés - les mouvances islamiste et marxisante - et ses localisations: le sud-est de la Turquie et l'Europe, où ces mouvements sont souvent actifs dans l'espace immigré. Deux partis clandestins, le PKK (Partiya Karkarên Kurdistan, Parti des Travailleurs du Kurdistan) et le Hizbullah, servent plus particulièrement de base à cette étude. Rappelons que le PKK est un mouvement nationaliste kurde d'inspiration marxiste constitué en 1978. En 1984, il lance une guérilla qui a entraîné plusieurs dizaines de milliers de morts et la mobilisation de centaines de milliers de militaires turcs. En 1999, l'arrestation d'Abdullah Öcalan, chef

2 Les Kurdes sont très majoritaires dans les régions du sud-est (Batman, Diyarbakır, Mardin, Şirnak, Siirt, Bitlis, Şanlıurfa, Muş, Van et Hakkari). Les provinces de Sivas, Maraş, Malatya, Elazığ, Erzincan, Tunceli et Bingöl sont marquées par un clivage alévi-sunnite et kurde-turc, alors que les provinces de Kars, Erzurum et Ağrı sont divisées selon un clivage linguistique (kurde-turc) et migratoire (migrants-tribus autochtones). (Bozarslan 1999). 
Dorronsoro, Gilles ; Grojean, Olivier (2004) 'Engagement militant et phénomènes de radicalisation chez les Kurdes de Turquie', European Journal of Turkish Studies, URL: http://www.ejts.org/document198.html To quote a passage, use paragraph (§).

de l'organisation depuis ses débuts, mettra fin à la lutte armée. Le parti vit depuis lors des lendemains incertains, entre déstructuration et consolidation. II a changé deux fois de nom (Kadek, puis Kongra-Gel) et espère toujours être accepté comme interlocuteur par les autorités turques et américaines ${ }^{4}$. Le Hizbullah, constitué à la fin des années 1980, appartient à la mouvance islamiste et milite pour un État islamique kurde 5 . Divisé en plusieurs groupes antagonistes (lilim et Menzil), il sera démantelé par une série d'opérations de police à partir de la fin des années 1990.

[6] A partir de ces deux groupes, notre étude vise à comprendre le processus de radicalisation à travers trois angles complémentaires. En premier lieu, les groupes radicaux étant généralement formés d'individus dépourvus de capital social et économique, la radicalisation peut donc s'analyser comme une stratégie pour accumuler des ressources collectives au sein d'un « espace de mouvement social» (Mathieu 2002). Celui-ci offre des cadres d'interprétation et des modes d'action à la jeunesse kurde des années 1970. La relation particulière à l'État - qui naît dans les interactions de la vie quotidienne et dans la mémoire des révoltes - structure également les répertoires d'action de ces organisations et notamment le recours à l'action violente (I). En second lieu, l'étude des trajectoires militantes des différentes cohortes d'adhésion (ensemble des individus qui s'engagent dans une même période) du PKK et du Hizbullah dans les régions kurdes et en Europe permettra de mieux comprendre comment s'effectuent les choix individuels d'engagement $(\mathrm{II})^{6}$. Enfin, l'adhésion à une organisation radicale questionne la notion d'habitus ${ }^{7}$. La capacité des acteurs à articuler des univers de sens souvent contradictoires sera analysée au regard des figures d'ambivalence qui permettent de devenir, mais aussi

3 De précédents terrains nous laissent penser que des analyses comparables peuvent être faites, notamment pour les islamistes afghans et l'UNITA.

4 Dans l'importante littérature sur ce parti, signalons les études de İsmet İmset (1992) et Paul White (2000).

5 Sur ce parti voir Ruşen Çakır (2000), Dorronsoro (2004). D'autres mouvements du même type sont également actifs, notamment le Kürdistan İslami Hareket fondé par un groupe d'oulémas kurdes en juillet 1993, sous la direction du leader spirituel Mollah Evdıllahe Xerzi Timoki et le Kürdistan İslam Partisi (Partiya islamiya Kürdistani) fondé le 11 décembre 1980 (Bulut 1994 : 667).

6 Nous analyserons deux cohortes d'adhésion au Hizbullah et trois cohortes d'adhésion au PKK. Pour l'Europe, seul le PKK est concerné, le Hizbullah n'ayant jamais reçu de soutien notable au sein de la diaspora.

7 Revenant à une conception plus nuancée, et probablement plus proche de celle de Max Weber, les travaux récents vont généralement dans le sens d'un acteur dont la socialisation implique un habitus pluriel et contradictoire. Pour une synthèse du débat voir Lahire (1998). 
Dorronsoro, Gilles ; Grojean, Olivier (2004) 'Engagement militant et phénomènes de radicalisation chez les Kurdes de Turquie', European Journal of Turkish Studies, URL: http://www.ejts.org/document198.html To quote a passage, use paragraph (§).

de rester militant ${ }^{8}$. Nous verrons que ces figures permettent de mettre en cohérence les ruptures biographiques et les dissonances cognitives, nées du passage d'un univers de sens à un autre (III).

\section{Espace des mobilisations et rapport à l'État : la constitution d'un groupe radical}

\section{Ressources et concurrence}

[7] Les individus qui sont à l'origine des mouvements radicaux kurdes dans les années 1970 ont un profil social et, notamment, une relation à l'État comparables. Alors même que le système scolaire s'est remarquablement développé durant les années $1960^{9}$, il n'offre que des perspectives limitées aux diplômés. On peut ainsi considérer sans trop forcer le trait que les militants fondateurs du PKK, pourtant en phase d'ascension sociale par rapport à leur milieu familial, sont tenus à l'écart des formations d'excellence des grandes universités ou des départements prestigieux et ont des perspectives d'emploi limitées dans une société encore largement rurale (Mardin 1978)10. Faute d'un secteur privé suffisant, l'arrivée de nombreux diplômés sur le marché du travail fait apparaître la difficulté à les employer. Dans le sud-est, le retard économique s'accroît et la main d'œuvre kurde, mal formée, commence à partir chercher du travail dans les métropoles de l'ouest du pays. Dans une société où l'éducation est valorisée, la jeunesse estudiantine est déçue par cette absence de perspective professionnelle, alors que le passage au lycée ou à l'université a produit une rupture qui rend difficile le retour dans le milieu initial. De ce point de vue, les biographies des leaders du Hizbullah (illim) et du PKK, Velioğlu et Öcalan, racontent en partie la même histoire (voir les biographies en annexe). Tous deux sont issus de milieux modestes, sans capital social ni économique. Après leur passage par la

8 Nous ne nous intéresserons pas ici au « maintien » de l'engagement mais uniquement aux dispositifs qui conduisent la jeune recrue à « se penser » dans l'organisation immédiatement après l'adhésion. Pour une approche en terme de carrières militantes, voir Bennani-Chraïbi, Fillieule (2003 : 118).

9 De 20000 étudiants en 1945 à 53000 en 1960.

10 Au Hizbullah, la plupart sont bacheliers, relativement peu sont passés par l'université (la principale exception est Velioğlu), ce qui est une différence importante avec le groupe initial du PKK. II y a cependant peu de véritables intellectuels au sein du PKK, à part peut-être Haki Karaer, qui meurt rapidement (White 2000 : 136). 
Dorronsoro, Gilles ; Grojean, Olivier (2004) 'Engagement militant et phénomènes de radicalisation chez les Kurdes de Turquie', European Journal of Turkish Studies, URL: http://www.ejts.org/document198.html To quote a passage, use paragraph (§).

Faculté de science politique d'Ankara, leur démarche est ambiguë - surtout dans le cas d'Öcalan, un temps proche de la droite nationaliste - et une intégration individuelle par le fonctionnariat les tente tous les deux ${ }^{11}$. Ce milieu de jeunes diplômés du secondaire ou de l'université est particulièrement favorable à l'action collective, en ce qu'il permet la concentration d'individus éloignés physiquement et socialement de leur milieu familial, et donc davantage disponibles aux entreprises de mobilisation des militants étudiants. Pour ces derniers, l'université devient rapidement le lieu d'une professionnalisation en politique. Ils n'ont pas initialement d'appui social, ce qui renforce l'idée de passage par la "Révolution», c'est-à-dire par un coup d'État. L'accès direct et non négocié aux ressources étatiques est un phantasme qui reflète la difficulté du groupe à entrer dans un rapport de négociation du fait de la faiblesse de ses moyens réels. La pensée des organisations radicales est centrée de façon obsessionnelle sur l'État, ce qui reflète à la fois l'orientation de l'enseignement (Copeaux 1997) et les idéologies dominantes du moment. Même chez les islamistes, le projet est d'accroître les ressources étatiques, notamment par des nationalisations. La perspective d'un coup d'État n'étant pas crédible, le projet peut basculer vers un contre-État, qui permet l'accumulation de ressources (biens économiques, appareil de coercition, etc.).

[8] La mobilisation des étudiants et lycéens dans une période d'extension rapide du système éducatif est un phénomène qui n'a rien d'inhabituel (Martin 1980). Ces groupes sont souvent dans une situation révolutionnaire au sens de Tilly (1978) en ce qu'ils demandent un accès élargi aux ressources étatiques. La radicalisation apparaît ici comme une stratégie de mobilisation - construite comme telle a posteriori par le chercheur - fréquente quand des individus à faible capital social et économique se constituent en acteur collectif.

11 La différence est nette avec Mansur Güzelsoy, le leader du Hizbullah Menzil. Né en 1948 dans le village de Zozınç (Başpınar), il suit les cours de l'école coranique locale comme tous les enfants. A 18 ans, il se marie, puis continue ses études religieuses dans différents villages autour de Diyarbakır. II restera fahri imam, imam non officiel, dans son village et n'aura jamais de poste officiel. 
Dorronsoro, Gilles ; Grojean, Olivier (2004) 'Engagement militant et phénomènes de radicalisation chez les Kurdes de Turquie', European Journal of Turkish Studies, URL: http://www.ejts.org/document198.html To quote a passage, use paragraph (§).

[9] La radicalisation apparaît cependant comme une stratégie orientée vers le groupe lui-même et non vers le reste de la société12. La théorie de l'avant-garde (islamiste ou communiste) joue ici un rôle particulièrement important : elle a pour effet pratique de se concentrer sur le noyau de l'organisation, sans s'intéresser à la réception du discours. Les frontières de l'appartenance au groupe se marquent en imposant des pratiques spécifiques (vestimentaires, pileuses, langagières etc.), comme autant de signaux qui renforcent la cohésion et l'homogénéité du collectif. L'engagement accru des militants compense la faiblesse des ressources individuelles. Les mouvements les plus radicaux se caractérisent alors par un mode d'organisation cloisonné et militaire qui substitue la solidarité de parti aux solidarités "naturelles" de la famille, et compense l'infériorité de leur position sociale. L'idéologie est vécue sur un mode radical et totalisant, jusque dans la sexualité de l'individu qui doit être contrôlée par l'organisation. La pureté du groupe, toujours à parfaire, permet exclusions et purges internes pour normaliser les comportements. Pour prendre un exemple inverse, les nationalistes kurdes davantage liés aux élites traditionnelles sont dans un rapport plus ambigu avec leur propre société et des formes d'intégration anticipées limitent la radicalité de ces militants. Le cas de Rizgari, une petite association nationaliste kurde, montre un investissement dans les activités culturelles et une moindre rupture avec le reste de la société malgré un langage tout aussi radical. Les militants ont ici un capital social plus important, certains sont fils de notables ou issus de la bourgeoisie urbaine des villes (Diyarbakır notamment, mais aussi Muş-Varto, Erzurum). Le culturel va devenir un lieu d'investissement (revue, maison d'édition etc.) qui joue contre la fermeture du groupe et favorisera l'insertion sociale des militants après le démantèlement du réseau suite au coup d'État de 1980. Leur capital scolaire et plus généralement culturel leur permet une mobilité sociale et géographique (dans l'ouest de la Turquie). Fréquentant des milieux intellectuels, ils nouent des liens avec la gauche turque, notamment syndicale, ce qui n'empêchent pas certains de continuer à s'engager dans la cause kurde, y compris dans la mouvance du PKK. Dans ce type de configurations, le degré de radicalité/fermeture est donc lié aux ressources initiales.

12 Alors même que la mobilisation est possible comme le montre la manifestation massive d'août 1967 à la suite d'articles anti-kurdes parus dans Otuken, une revue pan-touraniste. C'est la période où les militants kurdes tentent, finalement sans succès, de trouver une place au sein de la gauche turque. 
Dorronsoro, Gilles ; Grojean, Olivier (2004) 'Engagement militant et phénomènes de radicalisation chez les Kurdes de Turquie', European Journal of Turkish Studies, URL: http://www.ejts.org/document198.html To quote a passage, use paragraph (§).

[10] Le processus de radicalisation est également lié à la concurrence entre organisations à l'intérieur d'un même espace politique. Les différents groupes forment un «espace de mouvement social», relativement autonome des autres espaces politiques (Mathieu 2002), d'autant plus que, dans le contexte turc, nombre de mouvements tolérés ou clandestins ne peuvent pas participer au jeu politique légal13. La radicalisation passe alors par la lutte pour s'imposer comme seul représentant de la « cause » en soulignant les différences théoriques - souvent obscures - entre organisations et courants politiques. Mais, malgré son importance, le discours n'est pas fonction de la radicalité du groupe car, en pratique, les discours appelant à la révolution immédiate et à la subversion de l'ordre social sont communs à la plupart des mouvements et ne préjugent pas du degré de fermeture de l'organisation. Par ailleurs, le discours est souvent appuyé par la force : il ne s'agit pas d'un espace de discussion rationnel, mais d'un affrontement symbolique qui peut rapidement devenir physique.

\section{La formation des répertoires d'action : radicalité, rapport à l'État et violence}

[11] La radicalité implique-t-elle un répertoire ou des modes d'action déterminés ? Une des questions centrales que pose la radicalisation est en effet celle du lien avec la violence. II n'y a pas de relation immédiate entre le degré de radicalité et le passage à la violence. Malgré l'idée reçue d'un lien mécanique entre radicalité idéologique et passage à la violence, les modes d'action des mouvements analysés - comme la faction Menzil du Hizbullah et la plupart des groupes nationalistes kurdes - montrent que la violence est généralement utilisée de façon très ponctuelle. S'il existe bien une relation entre radicalité et violence, elle est indirecte, médiatisée par la relation avec l'État, qui est d'autant plus décisive que les revendications des groupes sont exclusivement dirigées vers ce dernier. Le passage à la violence peut ainsi s'analyser au regard de quatre éléments, qui tiennent tous à la relation avec l'État: les perceptions et a priori communs, les effets de la répression, la modification du contexte d'action et, dans le cas du Hizbullah, l'instrumentalisation directe.

13 Voir par exemple les statistiques officielles des incidents enregistrés en 1979-80, où Diyarbakır arrive après Istanbul, Ankara et Adana (Türkiye'deki...1982 : 210). 
Dorronsoro, Gilles ; Grojean, Olivier (2004) 'Engagement militant et phénomènes de radicalisation chez les Kurdes de Turquie', European Journal of Turkish Studies, URL: http://www.ejts.org/document198.html To quote a passage, use paragraph (§).

[12] La définition d'un répertoire d'action repose en premier lieu sur des a priori communs entre les adversaires. L'illégalité, qui est la situation habituelle de tous les mouvements nationalistes kurdes, ne peut expliquer à elle-seule l'émergence d'une stratégie de confrontation. Dans le cas des Kurdes, la longue tradition de violence étatique et de rébellion explique que les jeunes générations perçoivent souvent l'État comme un ennemi ${ }^{14}$. Certaines provinces du sud-est ont été sous état d'urgence entre 1978 et 2002 et, depuis le début de la République, les périodes d'administration normale ont duré moins longtemps que les états d'exception ou l'administration militaire. L'armée a appliqué dans le sud-est une stratégie de répression massive et indifférenciée parce qu'elle se représentait le "Kurdistan » comme un territoire ennemi, en raison notamment de la mémoire des révoltes des années vingt et trente. Or, dans les années 1970, la violence dans les régions kurdes n'est pas plus importante qu'ailleurs en Turquie ${ }^{15}$. De plus, contrairement à d'autres régions de Turquie, il ne s'agit pas d'une violence milicienne, définie par un début de territorialisation des mouvements et de fortes différences idéologiques appuyées sur des polarisations identitaires (alévis contre sunnites par exemple) ${ }^{16}$. Le lien entre engagement politique et appartenance communautaire ne fait donc pas sens à un niveau local (en dehors des marges des départements kurdes). Enfin, dans les années 1980 et 1990, comme encore aujourd'hui, une faible partie de la population souhaite l'indépendance; la plupart demandent une réforme de l'État pour mettre fin au sous-développement (Ergil 1995, CHP 1998). Cette « demande d'État » a été largement passée sous silence par les deux protagonistes car elle s'accorde mal avec leurs présupposés sur la crise. En dépit de ces éléments, il y a donc une curieuse

\footnotetext{
14 Les premières révoltes kurdes voient l'alliance des nationalistes kurdes qui se positionnent contre l'État turc, et des élites traditionnelles qui refusent l'État dans sa globalité (White 2000).

15 Les premières violences politiques des étudiants dans les années 1960 ne sont d'ailleurs pas fondamentalement différentes de ce qui se passe ailleurs en Europe dans cette période. L'élargissement du phénomène est dû à des causes complexes où l'attitude répressive du régime a une place centrale. On peut néanmoins se demander si les premières actions du PKK contre les grands propriétaires fonciers (Siverek, Batman) à la fin des années 1970 ne sont pas d'ores et déjà annonciatrices de la violence contre l'État. D'autres recherches s'avèreraient nécessaires, mais il semble possible de retracer les deux objectifs de cette violence. Elle est révolutionnaire et stratégique - peu différente de celle qui sera mise en œuvre contre l'État, mais c'est aussi, et surtout, une violence liée à la concurrence avec les autres groupes, les KUK (Kürdistan Ulusal Kurtuluşçular, Libérateurs nationaux du Kurdistan) notamment, l'idée du PKK étant de monopoliser les revendications kurdes.

16 Sauf précisément dans les « zones sensibles », à la marge des régions kurdes (Bozarslan 1999).
} 
Dorronsoro, Gilles ; Grojean, Olivier (2004) 'Engagement militant et phénomènes de radicalisation chez les Kurdes de Turquie', European Journal of Turkish Studies, URL: http://www.ejts.org/document198.html To quote a passage, use paragraph (§).

convergence entre l'armée et le PKK dans la perception du «Kurdistan» comme un territoire politiquement homogène et rebelle.

[13] Quels sont les effets de la répression sur la radicalisation ? II convient de dépasser à la fois la perspective psychologisante où la répression accentue la frustration et donc la mobilisation, et celle de la mobilisation des ressources qui considère au contraire la répression comme nécessairement dissuasive (Bennani-Chraïbi, Fillieule 2003 : 75). En fait, le contexte et la nature de l'organisation jouent ici un rôle essentiel en raison du rapport entre les effets de la répression et le degré de structuration des groupes (idem: 79). Par exemple, une majorité des militants nationalistes kurdes ont cessé d'avoir des activités politiques après le coup d'État et la répression de 1980. Ils appartenaient à des groupes qui n'étaient pas organisés pour résister à une répression dont ils n'avaient d'ailleurs pas envisagé l'ampleur : illégaux, ils n'étaient pas clandestins. L'exception est ici le PKK, plus structuré que les autres, et dont la direction avait déjà fuit en Syrie au moment du coup d'État. La prison a alors servi de réservoir militant au PKK comme au Hizbullah, leurs détenus y demeurant organisés ${ }^{17}$.

[14] Par ailleurs, les politiques des institutions peuvent créer (involontairement) un contexte favorable au passage à la violence. Ainsi, le coup d'État de 1980 et la répression qui s'ensuit ont permis l'établissement du monopole du PKK contre ses concurrents et son implantation dans les campagnes. Après la liquidation des autres groupes nationalistes kurdes, le PKK est en effet en face à face avec l'État, mais exclu des villes où la répression est trop efficace pour permettre son maintien. Immédiatement après le coup d'État, il ne parvient pas à obtenir d'aide dans la population rurale comme le montrent les récits des quelques militants encore actifs dans les campagnes en 1982-83. Or, l'oppression massive des populations kurdes se conjugue avec un retrait marqué des services de l'État dans les zones rurales, phénomène qui débute dans les années 1970 mais s'amplifie au début des années 1980. Ce changement de contexte provoque l'ouverture d'un espace politique pour le PKK dont le discours sur l'autodéfense des Kurdes devient crédible. Le lancement de la guérilla, en 1984 à partir de la Syrie, rencontrera ensuite un écho grandissant dans les régions kurdes. Le paradoxe est donc 
Dorronsoro, Gilles ; Grojean, Olivier (2004) 'Engagement militant et phénomènes de radicalisation chez les Kurdes de Turquie', European Journal of Turkish Studies, URL: http://www.ejts.org/document198.html To quote a passage, use paragraph (§).

que les politiques sécuritaires ont été le véritable moteur de l'implantation du PKK qui, d'un mouvement groupusculaire, est progressivement devenu une organisation légitime pour une partie importante des Kurdes.

[15] Enfin, dans un cas au moins, celui du Hizbullah Ilim, la manipulation est le facteur décisif de l'émergence de la violence à grande échelle. De nombreux documents permettent aujourd'hui de valider l'hypothèse d'un mouvement initial clandestin, mais peu violent, qui change de mode d'action à la fin des années 1980 sous l'impulsion d'une partie de l'appareil de sécurité, notamment certains services de la gendarmerie (JiTEM, Jandarma İstihbarat ve Terorle Mucadete) et les services secrets (MIT, Milli İsthbarat Teşkilatı) (Dorronsoro 2004). La branche lilim du Hizbullah pratique alors une violence orientée contre le reste du Hizbullah (la branche Menzil) et surtout contre le PKK. Les opérations contre les institutions d'État sont inexistantes jusqu'au moment où le gouvernement lance des opérations pour démanteler le mouvement, à la fin des années 1990.

\section{Les logiques de l'engagement : réseaux et dispositions}

[16] La situation décrite plus haut n'explique pas comment s'opère l'engagement, et encore moins l'engagement dans un groupe radical. Cette deuxième partie analyse donc les profils des fondateurs et des militants des deux mouvements dans les années 1980 et 1990, en montrant comment s'opère l'actualisation des dispositions qui conduit à l'engagement. Nous opposerons ici le groupe initial où les relations personnelles sont intenses et multidimensionnelles et les recrutements dans la deuxième et la troisième cohortes où l'organisation attire des profils plus diversifiés ${ }^{18}$.

\footnotetext{
17 Les prisonniers d'un même mouvement partagent la même cellule collective et participent à la formation politique de jeunes venus d'autres partis.

18 || faut ici signaler une différence de temporalité entre les deux mouvements. En ce qui concerne le PKK, on peut grosso modo établir quatre cohortes d'adhésion, qui correspondent assez bien à l'histoire du parti, même si des différences existent avec les Kurdes de Syrie, d'Irak ou d'Europe engagés dans le mouvement. La première cohorte est celle des fondateurs (1974-78) suivie par celle des débuts du mouvement (19791990). Cette dernière se divise en deux catégories : les premiers militants clandestins, souvent emprisonnés (1979-1984), puis les premiers guérilleros (1985-1990). La troisième cohorte est celle de l'élargissement du mouvement (1991-1998) et la quatrième correspond à ceux qui se sont engagés après l'arrêt de la lutte
} 


\section{Les fondateurs : des réseaux multiplexes}

[17] Dans le cas des fondateurs et des groupes initiaux, la multidimensionnalité des liens facilite la création de l'organisation. Les biographies des militants se recoupent à différents niveaux: même enseignement, même origine sociale et géographique, appartenance aux mêmes associations, liens familiaux, etc. II n'est pas rare de compter des fratries entières qui militent au Hizbullah, par exemple Çağıııcı, Polat, Varol, Mehmet Ali Bilici et Yaşar Polat (qui sont beaux-frères). Ceci a pour effet de faciliter le passage à la clandestinité car le groupe est déjà une société en miniature, en particulier un marché matrimonial et un milieu amical. Les leaders tendent à cumuler les appartenances à différents groupes de solidarité : famille/clan, lieu (au niveau du district ou de la province, surtout pour le Hizbullah), mais également associations, le passage par les mêmes organisations de jeunesse ou d'étudiants étant en effet décisif au sein des deux organisations.

[18] A Ankara, les futurs militants du PKK se regroupent au sein de l'AYÖD (Ankara Yüksek Öğrenim Derneği, Association des étudiants de l'université d'Ankara), alors proche du THKP-C. Ils ne sont alors qu'un groupe parmi beaucoup d'autres créés après le démantèlement de la gauche radicale turque (exécution de Mahir Çayan, Deniz Gezmiş) et du DDKO (Devrimci Doğu Kültür Ocakları), lors du coup d'État de 1971. Certains, dont Öcalan, ont déjà fait de la prison pour leurs activités militantes. Peu satisfaits de cette organisation, quelques-uns fondent en 1974 l'Ulusal Kurtuluş Ordusu (Armée de libération nationale), connue à partir de 1975 sous le nom de Kurdistan Devrimciler (Révolutionnaires du Kurdistan). Ils lancent des campagnes de propagande à Ankara, mais aussi à Antep, Diyarbakır et Elazığ, Batman, ralliant progressivement des soutiens. Les solidarités sont d'ores et déjà militantes et amicales, même si des solidarités familiales ou tribales sont également mobilisées quand le mouvement tente de s'implanter dans les villes kurdes à partir de 1978, date de fondation du parti. Dès les débuts, des Turcs de gauche et quelques militants d'origine arménienne (de Turquie) participent 
Dorronsoro, Gilles ; Grojean, Olivier (2004) 'Engagement militant et phénomènes de radicalisation chez les Kurdes de Turquie', European Journal of Turkish Studies, URL: http://www.ejts.org/document198.html To quote a passage, use paragraph (§).

également à la lutte. Les origines géographiques sont assez diverses. Les premiers militants, qui se retrouvent dans les mêmes universités, viennent de différentes régions kurdes. Avant 1980, l'origine géographique des fondateurs influence ainsi les zones de recrutement. Plus précisément, ce sont surtout des militants bien implantés localement, mobiles, qui participent ensuite à la diffusion du mouvement dans leur ville d'origine, d'abord chez les lycéens et étudiants, puis, de manière très progressive, dans les bourgs à partir de 1978'19. En 1982, la majorité des cadres - environ 300 militants professionnels clandestins - viennent des provinces de Mardin, Batman, Bingöl, Antep, Dersim et Urfa. II y a peu de cadres de Kars, Ağrı et Muş, et presque personne de Hakkari (Celik, 2002), peut-être en raison du faible nombre d'étudiants originaires de ces régions.

[19] La constitution du Hizbullah est en partie comparable. A partir des années 1950, différentes organisations, comme les Komünizmle Mücadele Dernekleri (Associations de lutte contre le communisme) réunissent à la fois des militants d'extrêmedroite (ülkücü) et des islamistes. La plupart des cadres de la première génération du Hizbullah sont marqués par cette socialisation conservatrice et anti-communiste, notamment au sein du MTTB (Milli Türk Talebe Birliği, Union nationale des étudiants turcs) et des Akıncılar Derneği (AK-DER, Association des éclaireurs), la branche jeunesse du Milli Selamet Parti, formée en 1976 et fermée en 1979. Ces deux organisations ne sont pas véritablement structurées à Batman et, pour les Akıncılar notamment, Istanbul est le lieu véritable des contacts. Un nombre significatif de militants se sont connus au MTTB : Hüseyin Velioğlu, Findan Güngör, Edip Gümüş, Bahrettin Özdemir, Abdülaziz Tunç (Faraç 2001: 118 ; Bulut, Faraç 1999: 79). Les Akıncılar sont également un point de passage commun à beaucoup de futurs militants Hizbullah dans les années 1970. En 1981, sept ou huit personnes issues des Akıncılar ont formé le İslami Hareket d'Istanbul, une branche indépendante, mais idéologiquement proche, du Hizbullah. Par ailleurs, Velioğlu, Altsoy, Yeşirlırmak, Şeyitoğlu, Hasan Dalgıç, Süleyman Dilek, Mehmet Ali Bilici ont tous été actifs au sein de l'Akıncılar Derneği. Cette association fournit notamment, dans le contexte de violence des années 1970, une formation paramilitaire aux militants. Par ailleurs, la

avec des militants géographiquement et socialement plus dispersés.

19 || ne s'agit donc pas principalement d'un recrutement dans les zones de tensions Kurdes/Turcs ou sunnites/alévis. 
Dorronsoro, Gilles ; Grojean, Olivier (2004) 'Engagement militant et phénomènes de radicalisation chez les Kurdes de Turquie', European Journal of Turkish Studies, URL: http://www.ejts.org/document198.html To quote a passage, use paragraph (§).

concentration géographique des premiers militants du Hizbullah met en lumière la faiblesse relative du recrutement étudiant, en comparaison avec les lycéens. De nombreux militants de la première génération sont de l'ilçe (arrondissement) de Gercüş : Velioğlu, Mehmet Ali Bilici, Süleyman Dilek, Şeşik Polat, et pratiquement tous sont originaires du département de Batman, y compris les militants basés à Istanbul (pour le İslami Hareket). La plupart des militants de Batman et notamment du district de Gercus appartiennent aux tribus locales (Sehinan, Kejan etc.), mais rien n'indique une adhésion collective où cette appartenance serait mobilisée comme telle. Enfin, un certain nombre d'organisations constituent le milieu commun de formation des premiers cadres du mouvement.

\section{Deuxième et troisième cohortes : la diversification des profils}

[20] Une fois l'organisation constituée, le recrutement se diversifie socialement, au moins dans le cas où le parti réussit à s'étendre, ce qui est le cas du PKK (des centaines de milliers de sympathisants, des dizaines de milliers de militants professionnels) et du Hizbullah (des milliers de militants) ${ }^{20}$. Dès lors, le recrutement se fait dans le cadre d'une organisation complexe, qui héberge différents profils de militants. En ce sens, l'organisation adapte son discours et celui-ci est également interprété de façon différente selon les individus. On voit alors jouer une double logique dans les recrutements : utilisation des réseaux existants (surtout villageois) et mobilisation des individus isolés, en rupture avec leur milieu, dans les grandes métropoles turques et européennes. Nous distinguons donc une série de situations selon le degré de médiatisation de la relation entre l'organisation et l'individu.

\section{Le recrutement au village}

[21] Le ralliement collectif de segments de population, par exemple des clans, est possible en milieu rural. Tout au long de son histoire, le PKK a ponctuellement utilisé la

20 Le milieu des années 1990 est probablement le moment du plus fort soutien populaire au PKK, qui dispose alors d'une véritable base sociale et militante. La même observation vaut probablement pour le Hizbullah, mais les données dont nous disposons sont très fragmentaires. 
Dorronsoro, Gilles ; Grojean, Olivier (2004) 'Engagement militant et phénomènes de radicalisation chez les Kurdes de Turquie', European Journal of Turkish Studies, URL: http://www.ejts.org/document198.html To quote a passage, use paragraph (§).

segmentation tribale pour se rallier une population (Hakkari, Siverek etc.). Le jeu de la segmentarité est complexe : dans certains cas, des clans travaillant pour le gouvernement au sein des korucu (milices) étaient proches de Mustafa Barzani (le leader du PDK d'Irak). De plus, le recrutement est souvent la conséquence des pressions gouvernementales : le clan ou le village sommé de choisir son camp doit devenir milicien ou assumer sa rupture et être le plus souvent vidé de sa population. Les ralliements collectifs sont néanmoins marginaux et ne permettent pas une véritable intégration des individus dans le mouvement.

[22] L'exemple d'un village au sud de Diyarbakır peut nous aider à comprendre comment les adhésions individuelles cette fois peuvent survenir dans une période de remise en cause des institutions villageoises. Sept clans, regroupés en deux groupes concurrents, représentent l'organisation de base du village ${ }^{21}$. Chacun va de préférence à sa mosquée et les deux groupes se regroupent respectivement à l'est et à l'ouest du village. Des vendettas entre familles renforcent l'appartenance au groupe. Les hommes mariés sont tenus de se rendre tous les jours aux réunions des sept oda (une par clan)22, ce qui les occupe pratiquement toute la journée en hiver. La politisation est inexistante avant la fin des années 1970. Les hommes politiques passent uniquement au moment des élections et parlent (en kurde) aux villageois, mais il n'y a pas véritablement de système clientéliste car ils n'offrent rien de très concret. Très peu de villageois, une dizaine sur plusieurs centaines de votants possibles, se déplacent pour aller voter. La pression démographique à partir des années 1960 va conduire à des migrations, au départ saisonnières, et à l'apparition d'une population très peu intégrée dans les structures villageoises qui perdent de leur centralité. La disparition des oda date des années 1970 ; l'appartenance au clan ne fait plus sens de la même manière dans les générations montantes. Les jeunes hommes et femmes (entre 15 et 20 ans) constituent dès lors une

21 En fait, Ahmet Ağa, le grand notable propriétaire d'une trentaine de villages dans la région a vu son influence diminuer nettement avec la République turque. II n'y a plus eu ensuite de liens très forts avec les villages environnants. Ce village est d'abord un regroupement de familles de conditions économiques assez peu différenciées au sein de sept clans.

22 Oda : la chambre, en fait la pièce réservée aux invités (séparée de la maison proprement dite où les femmes circulent). 
Dorronsoro, Gilles ; Grojean, Olivier (2004) 'Engagement militant et phénomènes de radicalisation chez les Kurdes de Turquie', European Journal of Turkish Studies, URL: http://www.ejts.org/document198.html To quote a passage, use paragraph (§).

population plus mobile géographiquement et moins intégrée économiquement et socialement au village qui ne représente plus nécessairement leur cadre de vie futur.

[23] Les militants PKK du village se recrutent dans ce milieu, apparemment sans exception. Dans ce cas, le militantisme, comme acte individuel, est rendu possible par un relâchement du contrôle clanique. Après 1984 et jusqu'à la fin des années 1990, le PKK fait sa propagande quand il passe dans les villages pour obtenir des vivres et des renseignements. La guérilla est soucieuse de ne pas heurter les villageois, distribue des cassettes de chansons kurdes et quelques journaux qui parlent de la première génération de militants martyrs. Les femmes guérilleros se voilent dans les villages (elles ne le sont pas dans les montagnes), ce qui facilite les contacts, tant avec les hommes qu'avec les femmes. Les combattants peuvent revenir quelques jours plus tard pour recruter des jeunes hommes et femmes impressionnés par l'efficacité et la discipline apparentes de l'organisation. L'engagement leur fournit l'occasion de s'éloigner du milieu familial sans rupture brutale avec la famille si elle soutient leur engagement ou d'échapper, par exemple, à un mariage arrangé. Les nouvelles recrues se sentent acteur d'un mouvement qui prétend puiser sa source dans les révoltes kurdes qu'elles connaissent par la tradition orale.

\section{La transmission de l'expérience militante}

[24] Pour certains jeunes, la mobilisation est directement liée à leur socialisation au contact de militants de gauche, kurdes ou turcs, dans les années 1970 (frères et sœurs plus âgées, oncles, professeurs). Le passage par la prison joue également un rôle extrêmement important. Dans les années 1980 et 1990, la prison fonctionne en effet comme un centre de formation pour le mouvement qui garde son emprise sur les militants détenus (Gürgoz 1997, Sevgat 2001). On devient même militant du PKK en prison en venant d'un autre groupe désorganisé par la répression. Pour d'autres, qui militaient souvent dans des organisations étudiantes ou lycéennes illégales (mais non clandestines), la prison constitue le lieu de l'engagement. II n'est d'ailleurs pas rare d'y rencontrer une partie de l'élite intellectuelle pro-kurde (İsmail Beşikçi par exemple) ou de côtoyer des militants fondateurs, d'autant plus que tous les nationalistes kurdes sont souvent 
Dorronsoro, Gilles ; Grojean, Olivier (2004) 'Engagement militant et phénomènes de radicalisation chez les Kurdes de Turquie', European Journal of Turkish Studies, URL: http://www.ejts.org/document198.html To quote a passage, use paragraph (§).

regroupés dans des cellules collectives d'une cinquantaine de personnes (la même chose est vraie pour le Hizbullah). Certains prisonniers relâchés dans l'attente de leur procès, sont dans l'impossibilité de rester dans leur ville. Surveillés par la police, ils se retrouvent souvent très seuls car ils sont soupçonnés avoir trahi sous la torture. Même s'ils n'étaient pas militants du PKK, certains s'engagent alors dans la guérilla ou gagnent l'Europe.

[25] L'émigration kurde de Turquie vers l'Europe date des années 1960. La politisation des Gastarbeiter s'accentue après le coup d'État de 1980, au moment où de nombreux réfugiés arrivent en Europe. Politisation et sensibilisation à la cause kurde ne signifient cependant pas engagement et, de fait, comme en Turquie, les années 1980 voient assez peu d'adhésions, sauf par exemple pour une partie des militants de Dev-Sol qui passeront au PKK. Au début des années 1990, un nombre beaucoup plus important de migrants de la deuxième génération adhérent au PKK, même si les primo-arrivants continuent de jouer un rôle moteur au sein du mouvement durant cette décennie. Deux cohortes - ceux déjà engagés en Turquie et parfois socialisés au sein du PKK et ceux politisés en Europe à partir des années 1980 - se retrouvent donc simultanément à un moment dramatique du conflit. Des jeunes de la seconde et troisième génération de migrants, collégiens ou étudiants, côtoient des jeunes primo-arrivants de la seconde cohorte d'adhésion, elle-même ayant souvent été en contact avec les premiers militants par la prison. La transmission de l'expérience militante s'en trouve ainsi fortement facilitée. Comme dans le cas des recrutements en Turquie, les relations familiales et les solidarités villageoises jouent un rôle essentiel dans les processus d'engagement. On accueille un militant clandestin originaire du même village, on apprend lors d'une manifestation l'histoire d'un ami tué dans les montagnes, on reçoit un cousin déjà engagé en Allemagne, qui parle de la situation des Kurdes dans son pays d'accueil et de la guérilla, ce qui permet une rapide prise de contact. L'interprétation politique d'expériences familiales traumatiques, la progressive extension de la répression en Europe, de même que certaines situations d'isolement (voir plus bas), pourront alors permettre un engagement vécu sur le même mode que celui de la deuxième cohorte de militants engagés en Turquie (Grojean à paraître). 
Dorronsoro, Gilles ; Grojean, Olivier (2004) 'Engagement militant et phénomènes de radicalisation chez les Kurdes de Turquie', European Journal of Turkish Studies, URL: http://www.ejts.org/document198.html To quote a passage, use paragraph (§).

Le recrutement d'individus isolés, en milieu urbain et en exil

[26] Le recrutement d'individus isolés est une constante des deux mouvements. Le Hizbullah est d'abord né dans un contexte social particulier, celui de la ville de Batman. Cette ville, à la fin des années 1970, a des spécificités qui peuvent expliquer l'émergence et l'implantation du mouvement ${ }^{23}$. Batman s'est en effet très rapidement développée en raison du pétrole, qui génère un revenu important. La raffinerie locale (Petrol İş), la principale industrie locale, a notamment assuré le développement du travail salarié. Du fait peut-être de son caractère récent et de l'origine villageoise des habitants, il y n'y a pas de culture urbaine, même si les choses changent aujourd'hui. Les lieux de sociabilité sont rares, et pratiquement inexistants pour les femmes. En comparaison avec d'autres villes proches comme Diyarbakır, les relations de voisinage paraissent limitées. Les syndicats et les associations fonctionnent, mais les individus participent assez peu à des actions collectives. La bourgeoisie de Batman ne constitue pas un groupe et n'a qu'un impact limité sur la vie sociale. Enfin, avec le développement de la guérilla dans les villages environnants, les institutions étatiques ont été en repli marqué dans les années 1980 et 1990, avec notamment un déficit d'imams et d'instituteurs. Les fonctionnaires (notamment les instituteurs) forment d'ailleurs une partie significative des militants (Dorronsoro 2004). Le soutien d'une partie de l'appareil de sécurité au Hizbullah a aggravé la crise de crédibilité des institutions. Dans ce contexte, la déstructuration des rapports sociaux dans les années 1980 et 1990 permet l'apparition de ce mouvement urbain qui recrute dans un prolétariat issu de l'exode rural, souvent forcé en raison des combats ${ }^{24}$. Le recrutement d'individus isolés récemment urbanisés est une caractéristique du Hizbullah qui n'a jamais, contrairement au PKK, eu d'enracinement rural. De plus, cette organisation est en phase avec l'individualisation du rapport au religieux, qui ne passe plus forcément par l'intermédiaire du mollah de village ou du fonctionnaire de l'administration des cultes (Diyanet).

23 || faut préciser que Batman a une image particulière, notamment du fait de la couverture médiatique des suicides de jeunes femmes en 1999 qui a répandu l'image d'un ville réactionnaire, très peu ouverte à la modernité.

24 D'où aussi le recrutement d'enfants. Par exemple, Lezgin Cangir rejoint l'organisation à quatorze ans. Responsable de l'équipe sorgu ve infaz (interrogatoire et exécution) de Diyarbakır, quinze morts et de cinq blessés lui sont attribués entre 1992 et 1997. 
Dorronsoro, Gilles ; Grojean, Olivier (2004) 'Engagement militant et phénomènes de radicalisation chez les Kurdes de Turquie', European Journal of Turkish Studies, URL: http://www.ejts.org/document198.html To quote a passage, use paragraph (§).

[27] Le recrutement du Hizbullah (lilim et Menzil) se fait d'abord au cours d'activités de prêche (tebliğ), dans les librairies, les cafés, les écoles professionnelles etc. Par exemple, l'action dans les mosquées permet d'avoir accès aux défavorisés, aux familles par le biais des enfants et sous prétexte de collecter des dons et opère une première sélection en permettant facilement le repérage des individus pratiquants. L'importance du travail dans les mosquées s'explique par l'effondrement des services publics dans le SudEst. Un rapport du Parlement turc de 1995 donne par exemple des chiffres sur le manque d'imams et la pénétration du Hizbullah, notamment à Batman. Dans cette ville, on estime en effet que sur 60 mosquées, 40 sont sous influence du Hizbullah (TBMM 1995: 113). D'après une déclaration du vali de Diyarbakır, le Hizbullah lim recrutait entre 1994 et 1996 dans 60 ou 70 mosquées à Diyarbakır (Çitlioğlu 2001: 176)25. Le noyautage des mosquées passe généralement par de bonnes relations avec les imams officiels, ou l'installation d'imam officieux quand les postes sont vacants, ce qui permet l'organisation de réunions politiques après la prière du soir. Les militants infiltrés s'occupent de l'organisation de cours, de pique-niques, de visites de cimetières, d'activités sportives. De plus, la plupart des cadres deviennent enseignants bénévoles pour donner des cours : cüz (étude de chacun des trente fascicules du Coran), tefsir (commentaire), langue arabe, tecvid (lecture du Coran), kültür elemanları (histoire religieuse). Ces cours en petits groupes favorisent la connaissance des individus et leur recrutement. Dans la deuxième génération de cadres, le recrutement dans les mosquées est fréquent. Cemal Tutar, Fuat Balca, Abdülkerim Kaya, Mustafa Ipek etc. ont tous été recrutés dans des mosquées et y ont souvent assuré des cours par la suite26.

[28] La deuxième et, surtout, la troisième cohorte de militants urbanisés du PKK est socialement beaucoup plus hétérogène que la première, en raison notamment de l'élargissement spectaculaire de la mobilisation et de la répression. Les villes du sud-est de la Turquie connaissent en effet une véritable explosion démographique liée à

25 Le groupe Menzil recrutait principalement dans une soixantaine de mosquées, notamment Berhampaşa et Hoca Ahmet Paşa (Batman), Başak Camii (Diyarbakır). Le llim était présent notamment dans les mosquées İskenderpaşa, Şehitlik, Bağlar, 5 Nisan, Lalabey, Alipaşa. On trouve une liste non exhaustive chez Bulut Faraç (1999: 213).

26 On se fonde ici notamment sur les biographies utilisées dans les réquisitoires lors des procès Hizbullah (T.C. Diyarbakır Devlet Güvenlik Mahkemesi Cumhuriyet Başsavcilığı, 2000). 
Dorronsoro, Gilles ; Grojean, Olivier (2004) 'Engagement militant et phénomènes de radicalisation chez les Kurdes de Turquie', European Journal of Turkish Studies, URL: http://www.ejts.org/document198.html To quote a passage, use paragraph (§).

l'évacuation de milliers de villages. Les paysans fraîchement arrivés en ville, touchés de plein fouet par le chômage massif et l'absence de scolarisation (par exemple à Diyarbakır), formeront la majorité des participants aux insurrections populaires (serhildan) de 1991 et 1992. Pour certains, cette mobilisation se prolongera par un engagement dans la guérilla. De même, en Europe, la mobilisation au sein du mouvement est parfois rendue possible par l'isolement des primo-arrivants, confrontés à des problèmes d'autorisation de séjour, de langue et de chômage. Le statut de réfugié est de plus en plus difficilement obtenu, les nouveaux migrants qui arrivent massivement ne sont pas toujours en position de s'intégrer à des réseaux. Devenir militant professionnel constitue alors parfois une réponse simple à l'isolement : pour peu que l'on ait été déjà sensibilisé à la cause en Turquie, s'engager fait passer de l'illégalité excluante à la clandestinité gratifiante, du chômage à l'hyper-activité militante. L'association locale est également un lieu de sociabilité (manifestations, fêtes, réunions ou simplement café-restaurant-coiffeur) qui a des effets importants sur la socialisation primaire et secondaire des individus, jusqu'à servir régulièrement de pont avec la guérilla. Une règle tacite voulait en effet que les familles kurdes, parfois sous la contrainte mais le plus souvent de bon gré, envoient un de leurs enfants dans les montagnes. Les camps de jeunesse du mouvement, où sont enseignés à la fois la théorie politique et le maniement des armes, ont constitué un premier palier dans l'engagement radical. Les jeunes pouvaient alors partir dans les montagnes kurdes ou s'engager dans les organisations de jeunesse en Europe.

\section{L'articulation des univers de sens}

[29] Dans cette troisième partie, nous abordons la dimension cognitive de la radicalisation. L'appartenance à un groupe radical suppose souvent d'adhérer à un univers de sens différent de la socialisation initiale ${ }^{27}$. Des villageois, sans éducation scolaire, évoluant dans un univers où l'islam et l'honneur (namus) jouent un rôle central, adhèrent à un parti marxiste-léniniste athée, qui prône le collectivisme et la libération de la femme.

\footnotetext{
27 La rupture peut être relativisée même pour un groupe radical, au moins quand celui-ci se développe et se complexifie, par exemple avec l'apparition d'une aile légale. L'engagement peut alors être modulé dans son intensité, du soutien financier à la guérilla.
} 
Dorronsoro, Gilles ; Grojean, Olivier (2004) 'Engagement militant et phénomènes de radicalisation chez les Kurdes de Turquie', European Journal of Turkish Studies, URL: http://www.ejts.org/document198.html To quote a passage, use paragraph (§).

Des militants européens du PKK, issus parfois de familles économiquement et socialement « intégrées », ont également basculé dans un autre univers de sens. L'islam du Hizbullah est en rupture avec celui du Diyanet et des villages, par son accent politique, son rejet des hiérarchies sociales et de l'État. Quelles sont les conséquences de ce passage au niveau individuel?

\section{La décision d'adhésion : spontanéité et distanciation}

[30] Si une partie des militants lycéens ou étudiants ayant déjà été sensibilisés à la cause se sont engagés spontanément dans le mouvement sur le mode de l'imaginaire de la lutte armée, on observe également chez de nombreux militants éduqués une phase d'isolement parfois relativement longue (quelques mois) juste avant l'adhésion. Cet isolement peut être la conséquence d'un deuil, d'une scolarité à la ville alors que la famille est restée au village, d'un emprisonnement, d'une fuite ou d'une migration, cumulé parfois à un état dépressif. Durant cette période, les futurs militants vont généralement procéder à une remise en cause de l'ordre social qu'ils avaient jusque-là intériorisé (hiérarchie familiale, sexuelle, étatique). Contrairement aux militants d'origine modeste recrutés en milieu rural, le conflit de génération est ici central, souvent dramatique. Ce processus est le plus souvent inconscient, mais il arrive, comme pour une conversion religieuse recherchée, de se couper volontairement du monde afin de « répondre aux questions que l'on se pose ». En fait, la réponse passe le plus souvent par la déconstruction au moins partielle des schèmes cognitifs qui sous-tendent son rapport au monde. Pour les moins éduqués, souvent de la deuxième et de la troisième cohorte, recrutés dans les villages ou dans les quartiers périphériques des grandes villes, le processus apparaît sensiblement différent et semble souvent être guidé par la saisie de l'opportunité. Si les militants peuvent avoir vécu une phase de questionnements similaire, la rupture avec le milieu d'origine est plus nette, moins intellectualisée. Elle est aussi vécue davantage sur le mode de la " tradition des révoltes », la répression favorisant le désir de " partir protéger la famille ». Chez les militants du Hizbullah, on a vu enfin que l'anonymat urbain favorisait également l'engagement, alors que pour la première génération des fondateurs du Hizbullah ou du PKK, c'est au contraire la dynamique de groupe qui joue. 
Dorronsoro, Gilles ; Grojean, Olivier (2004) 'Engagement militant et phénomènes de radicalisation chez les Kurdes de Turquie', European Journal of Turkish Studies, URL: http://www.ejts.org/document198.html To quote a passage, use paragraph (§).

[31] L'engagement semble donc favorisé par des situations conjoncturelles de crise des croyances. Les univers de sens sont contestés et ne permettent plus une cohérence des représentations du monde. Or, dans ces situations, la simplification est recherchée, si elle permet une mise en récit, un gain de cohérence des évènements traumatiques. L'univers de sens se construit collectivement, mais produit une recomposition des habitus individuels. Les tensions qui résultent des incohérences de I'habitus peuvent notamment se résoudre par deux processus. En premier lieu, l'investissement affectif et intellectuel sur les figures d'ambivalence culturelle - qui fonctionnent dans les deux univers - facilite le passage et atténue les tensions psychologiques. La position de recul a préalablement favorisé la remise en question de son environnement et de soi-même. En second lieu, la discipline et la reconstruction biographique sont productrices de sens : elles mettent en récit la rupture et permettent de réinterpréter le passé pour l'expliquer par le présent. On est dans un mécanisme de conversion assez classique que l'on retrouve dans les hagiographies religieuses.

\section{Les figures d'ambivalence}

[32] La première figure d'ambivalence se rencontre sous la forme du mythe. Les mythes politiques sont ici compris comme des récits collectivement produits qui permettent d'organiser le monde. Donnant un sens au présent, reliant le passé à l'avenir dans une vision téléologique du monde, il participe de la définition des identités en marquant la frontière entre "ceux qui y croient et ceux qui n'y croient pas " (Grojean ; Küçük à paraître). On observe en fait chez la plupart des militants rencontrés à la fois un rejet violent des mythes politiques turcs et une adhésion inconditionnelle aux mythes politiques kurdes, alors même que ces mythes sont construits et pensés sur le même mode (origine, théorie de l'homme nouveau, leader national, idée de l'action révolutionnaire). Cette structure comparable du récit peut faciliter le passage d'un discours à l'autre. L'institution scolaire turque, vecteur essentiel du kémalisme, est également rejetée bien que les militants tendent à reproduire les modes de pensée enseignés à l'école.

[33] Le charisme est le deuxième élément de l'ambiguité, qui permet de rallier des soutiens individuels ou collectifs. Le « charisme d'organisation » qu'il faut ici différencier de 
Dorronsoro, Gilles ; Grojean, Olivier (2004) 'Engagement militant et phénomènes de radicalisation chez les Kurdes de Turquie', European Journal of Turkish Studies, URL: http://www.ejts.org/document198.html To quote a passage, use paragraph (§).

la catégorie wébérienne, a pour principale caractéristique d'être construit par un parti. Le chef est à la fois l'expression d'une organisation totale, qui tend à politiser l'ensemble des domaines de la vie sociale, et peut être aussi le réceptacle d'un investissement affectif qui reprend des catégories existantes comme celle de la parenté. Öcalan est "Apo » (diminutif d'Abdullah), un oncle, un père, précisément sur le modèle d'Atatürk (le père des Turcs). A un autre niveau, le chef charismatique peut être une incarnation du rebelle/bandit qui a une place centrale dans l'imaginaire kurde. Les chansons consacrées à la guérilla vont dans le sens d'une continuité entre le PKK et les mouvements de révolte précédents. La mise en scène tragique ou parfois comique reprend les thèmes du brigand d'honneur opposé à l'État. En permettant une double lecture du leader, le charisme fonctionne comme un compromis ambigu entre les attentes de l'organisation et celles de la population. En fait, dans une organisation très structurée qui a un projet politique et de politisation, le charisme du chef rend le message compréhensible car réinterprété selon des catégories plus acceptables (la thématique du bon chef) qui permettent pour la population de court-circuiter en partie les procédures de l'organisation (Dorronsoro 2000 : 146). C'est un phénomène qu'il faut néanmoins replacer dans une chronologie particulière : la figure d'Öcalan est déjà centrale au sein du mouvement dès les années 1970 - on surnomme les militants "Apocular », partisans d'Apo -, mais ce n'est qu'à la fin des années 1980 et au début des années 1990 que le charisme d'Öcalan opère véritablement au sein de la société kurde en Europe et en Turquie. Si, dans les années 1980 (deuxième cohorte), on s'engage dans une organisation marxiste et nationaliste, dans les années 1990 (troisième cohorte), on s'engage massivement dans l'armée d'Öcalan : le charisme d'organisation semble ainsi progressivement se transformer en se diffusant dans de larges pans de la société. Là encore, des différences significatives sont à noter entre les groupes urbains éduqués et les populations rurales, ou entre les générations. Le phénomène charismatique dépasse malgré tout très largement l'appartenance à un groupe social. L'idéologie marxiste-léniniste ne compte alors plus réellement: sommé de choisir son camp, on s'engage d'autant plus pour Öcalan qu'aucune option légale crédible n'est proposée par le régime turc jusqu'au milieu des années 1990. Le cas du Hizbullah est différent en ce que la nature très clandestine du mouvement fait que Velioğlu n'est 
Dorronsoro, Gilles ; Grojean, Olivier (2004) 'Engagement militant et phénomènes de radicalisation chez les Kurdes de Turquie', European Journal of Turkish Studies, URL: http://www.ejts.org/document198.html To quote a passage, use paragraph (§).

pratiquement pas connu à l'extérieur ; le charisme ne fonctionne que dans l'organisation, par rapport aux militants 28 .

[34] Une dernière figure que l'on peut signaler est le namus, qui est réinterprété dans le cadre du parti. Le contrôle des femmes, détentrice de l'honneur de la famille, se transforme en protection des militantes par les amis militants du frère par exemple quand celui-ci est dans les montagnes ou en prison. La solidarité de parti fonctionne sur le même mode que les solidarités de famille, et beaucoup de mariages se font entre militants (pour le Hizbullah29), ce qui accroît la confusion entre les deux systèmes de valeurs. De même, les faits d'armes sont valorisés au nom de la lutte, mais on peut aussi y lire en écho un rappel des valeurs de bravoure personnelle, constitutifs de la renommée individuelle.

\section{La discipline et l'exercice biographique}

[35] Si les figures d'ambivalence permettent ainsi de servir de pont entre les deux univers, elles n'expliquent pas comment l'engagement tend à s'inscrire dans la durée. Les ruptures traumatiques créent une difficulté à se penser comme un individu cohérent, d'où plusieurs types de réaffirmation de soi. La prise en compte des « effets que le groupement lui-même a sur l'individu engagé » (Sawicki 2003) exige alors d'analyser comment l'acteur met en conformité son habitus avec le nouvel univers dans lequel il évolue. La discipline et la réécriture biographique, dans un contexte de clandestinité à hauts risques, sont deux éléments qui vont permettre de mettre plus durablement en cohérence les trajectoires militantes.

[36] La discipline militaire permet tout d'abord de dresser les corps, d'organiser le temps public et privé dans l'unique perspective de la révolution, d'aplanir les différences culturelles entre militants de différentes couches sociales et surtout d'obtenir l'obéissance. Elle passe par une stricte hygiène de vie (interdiction des relations sexuelles, des boissons alcoolisées et des drogues), par des rituels d'allégeance et par l'autocritique, qui est un

\footnotetext{
28 Velioğlu est mentionné dans la presse pour la première fois le 16 février 1992 dans 2000'e Doğru et sa photo publiée dans le même magazine un an après.

29 Le mariage est strictement interdit chez les militants du PKK, phénomène que l'on retrouve dans différents mouvements de guérilla, l'UNITA notamment.
} 
préalable efficace à la réécriture biographique. Cette discipline est le plus souvent considérée comme légitime: la fidélité à Öcalan et les risques encourus vont dans le même sens que l'idéologie de l'« homme nouveau » qui implique de renoncer à son ancienne personnalité pour devenir un « vrai Kurde » (White 2000 : 138). La discipline est également souvent vécue sur le mode de la reconstruction de soi après la phase d'isolement et de désorientation préalable à l'adhésion. II reste qu'un certain nombre de jeunes recrues engagées depuis peu dans la guérilla et croyant que les montagnes étaient synonymes de 'liberté' ont ainsi fait l'amère expérience d'une discipline du corps et de l'esprit incompatibles avec leur projet personnel. Des désertions ont donc régulièrement lieu au cours de la formation initiale ou parfois au bout de quelques années. Ainsi, sur un groupe d'une soixantaine de femmes en formation de 3 mois dans le nord de l'Irak au premier semestre 1997, il pouvait y avoir jusqu'à deux départs clandestins par jour. L'emprisonnement ou l'exécution pouvaient sanctionner les désertions. Des formes de dépression affichées permettent aussi de sortir sans rupture de la guérilla et de (re-)partir en Europe. La prise en charge totale des individus par le parti et la violence interne exercée sur les réfractaires oblige néanmoins bien souvent - en cas de désaccord - à tenter d'harmoniser sa propre vision du monde à celle du parti.

[37] C'est par un travail autobiographique que l'acteur justifie ensuite son engagement, justifications qu'il faut évidemment appréhender comme constructions en vue d'une mise en cohérence personnelle et non comme des déterminants de l'engagement. Au moins d'un point de vue analytique, on peut faire la différence entre les biographies produites directement par le parti (notamment les nécrologies dans les périodiques liés au mouvement) et les exercices plus personnels où le militant s'exprime directement (mémoires, journal de bord, entretien avec le chercheur). Dans les deux cas, la vie avant la conversion prend un sens par rapport à celle-ci (thèmes notamment des signes annonciateurs). La reconstruction permet de considérer que malgré la rupture c'est en fait un aboutissement logique, une trajectoire normale. Toute sortie du parti, en dehors d'une démobilisation générale, sera ensuite difficile pour le militant: un sens a été donné à sa vie, il est coûteux psychiquement et socialement de se « réécrire » à nouveau. 
Dorronsoro, Gilles ; Grojean, Olivier (2004) 'Engagement militant et phénomènes de radicalisation chez les Kurdes de Turquie', European Journal of Turkish Studies, URL: http://www.ejts.org/document198.html To quote a passage, use paragraph (§).

\section{Conclusion}

[38] Cette tentative de réflexion sur la radicalisation ne prétend pas, c'est une évidence, à une validité générale, même si des comparaisons peuvent être faites entre la France, l'talie et la Turquie pour ce qui est de la radicalisation des groupes étudiants et leur marginalisation dans la plupart des cas (notamment le DHKP-C). Dans le cas kurde, la faiblesse du capital social paraît être une constante parmi les fondateurs des groupes radicaux, pour lesquels une mobilisation militarisée permet l'accumulation de ressources politiques. D'autre part, la fermeture brutale du système politique apparaît comme une condition de l'élargissement de l'audience du mouvement. En effet, une rupture politique importante (répression généralisée de 1980 à 1983) a incité la population à soutenir un mouvement radical, alors même que le coup d'État de 1980 était au départ perçu positivement (en raison des violences des années 1970). Les seuls groupes radicaux qui n'ont pas été marginalisés en Turquie sont en effet kurdes, un espace où la politique répressive de l'État a longtemps créé une zone de non-droit. En second lieu, l'étude diachronique des différentes cohortes d'adhésion au PKK et au Hizbullah, et notamment la distinction entre les réseaux aux liens multiplexes initiaux et les adhésions de la deuxième cohorte, a permis de restituer l'hétérogénéité des contextes d'engagement. Enfin, la radicalisation des individus nécessite une mise en cohérence de soi après l'adhésion. Les figures d'ambivalence que nous avons relevées ont ainsi montré comment le militant pouvait articuler des univers de sens souvent contradictoires.

[39] || resterait alors effectivement à s'intéresser au « maintien de l'engagement » ou au "désengagement» (Bennani-Chraïbi, Fillieule 2003 p. 118 ss $^{30}$ ), tout en questionnant les processus de "déradicalisation». Si ces questions dépassent les limites de cet article, il nous semble possible de proposer quelques pistes qui sont autant de projets de recherche futurs. Comme nous l'avons vu, un certain nombre de facteurs permettent d'abord de se penser "en phase » avec une organisation radicale (clandestinité et coercition du groupe, réécriture biographique qui permet la mise en cohérence des itinéraires et des destins militants). Les diverses rétributions associées à

\footnotetext{
30 Les auteurs distinguent quatre types de destins militants : la cooptation par le régime, le désengagement et le retrait dans des formes plus ou moins légales d'accommodation, la reconversion dans d'autres sphères, le retrait pur et simple de toute activité contestataire.
} 
Dorronsoro, Gilles ; Grojean, Olivier (2004) 'Engagement militant et phénomènes de radicalisation chez les Kurdes de Turquie', European Journal of Turkish Studies, URL: http://www.ejts.org/document198.html To quote a passage, use paragraph (§).

l'engagement, la professionnalisation des militants, de même que la complexification de l'organisation jouent assurément un rôle important dans le maintien de l'engagement. D'autre part, un certain nombre de reconversions permettent parfois de rester dans la mouvance du parti, tout en reprenant son autonomie : c'est par exemple le cas d'un certain nombre de militants réfugiés en Europe, qui, après avoir travaillé quelques années pour le parti, s'en distancient quelques peu afin de reprendre des études. Mais si ce phénomène semble davantage accepté pour le PKK depuis 1999, il était particulièrement difficile de quitter le parti avant l'arrestation d'Öcalan : une sortie du mouvement signifiait dans tous les cas le désengagement de toute activité militante.

[40] A un niveau plus collectif, les responsables de l'organisation peuvent être conduits à radicaliser le mouvement pour le renforcer ou au contraire à jouer l'ouverture pour capitaliser une popularité et faciliter une reconversion. Le rapport entre élargissement de l'audience et abandon de la radicalisation est en fait complexe. L'élargissement de son audience a conduit le PKK (et de ce qui est souvent considéré comme son aile légale, le HADEP) à une modération progressive (à partir du début des années 1990). On peut alors contester le fait que les phénomènes de martyre (immolations par le feu, attentatssuicides) aillent forcément de pair avec une radicalisation des organisations. II pourrait y avoir plutôt dé-radicalisation de l'organisation et radicalisation des militants, ce qui pose la question de l'évolution divergente de l'organisation et des militants. La radicalité des militants peut enfin survivre à l'organisation et conduire au réveil de réseaux dormants. Les attentats d'Istanbul ont montré que les anciens militants du Hizbullah avaient gardé une logique de radicalisation qui a survécu au parti, largement démantelé après les opérations de police de l'année 2000. Certains militants européens du PKK paraissent également s'inscrire dans ce schéma : si l'arrestation d'Öcalan a conduit à une déradicalisation sans précédent du mouvement, la reprise de la guérilla en 2004 semble encore aujourd'hui bénéficier d'importants soutiens en Europe. 


\section{References}

Apocular (P.K.K) Iddianame, (1981), Ankara, Gnkur.Basimevi.

Bennani-Chraïbi, Mounia ; Fillieule, Olivier (dir.) (2003) Résistances et protestation dans les sociétés musulmanes, Paris, Presses de Sciences Po.

Bozarslan, Hamit (1999) 'Le phénomène milicien. Une composante de la violence politique dans la Turquie des années 1970', Turcica, XXXI, pp. 185-244.

Bulut, Faik ; Faraç, Mehmet (1999) Kod Adı : Hizbullah. Türkiye Hizbullah'nın Anatomisi, Istanbul, Ozan Yayıncllik.

Çakır, Ruşen (2001) Derin Hizbullah, İstanbul, Siyahbeyaz, Metis Güncel.

Çelik, Selahattin (2002) Den Berg Ararat versetzen. Die politischen, militärischen, ökonomischen und gesellschaftlichen Dimensionen des aktuellen kurdischen Aufstands, Frankfurt am Main, Zambons Verlag.

Çittioğlu, Ercan (2001) Tahran-Ankara Hattında 'Hizbullah', Ümit Yayınclık.

CHP (Cumhuriyet Halk Partisi) (1998) Doğu ve Güneydoğu Ön Rapor, non-publié.

Copeaux, Etienne (1997) Espaces et temps de la nation turque, Paris, CNRS Editions.

Dorronsoro, Gilles (1999) 'L'islam kurde dans le sud-est de la Turquie', Les Annales de l'Autre Islam, nº, p. 115-134.

Dorronsoro, Gilles (2000) La révolution afghane, Paris, Karthala.

Dorronsoro, Gilles (2001) 'Les Kurdes de Turquie: revendications identitaires, espace national et globalisation', Les Etudes du CERI, $n^{\circ} 62$.

Dorronsoro, Gilles (2004) La nébuleuse Hizbullah, Istanbul, Dossiers de l'IFEA.

Ergil, Doğu (1995) Doğu Sorunu, Istanbul, TOBB.

Faraç, Mehmet (2001) Batman'dan Beykoz'a Hizbullah'ın Kanlı Yolculuğu, Günizi Yayıncllık, novembre.

Grojean, Olivier (à paraître) 'Immigration et solidarités transnationales : l'engagement politique en exil', in Devin, Guillaume (dir.) Les solidarités transnationales aujourd'hui, Paris, L'Harmattan.

Grojean, Olivier ; Küçük, Bülent (à paraître) 'Les recompositions du pouvoir au sein du mouvement national kurde de Turquie', Etudes kurdes, $n^{\circ} 7$.

Gürgoz, Ali Ekber (1997) La nuit de Diyarbakir, Paris, L'Harmattan.

Imset, İsmet G. (1992) The PKK. A Report On Separatist violence in Turkey, Ankara, Turkish Daily News Publications.

Lahire, Bernard (1998) L'homme pluriel, Paris, Nathan.

Mardin, Şerif (1978) 'Youth and Violence in Turkey', Archives européennes de sociologie, XIX, pp. 229-254. 
Dorronsoro, Gilles ; Grojean, Olivier (2004) 'Engagement militant et phénomènes de radicalisation chez les Kurdes de Turquie', European Journal of Turkish Studies, URL: http://www.ejts.org/document198.html To quote a passage, use paragraph (§).

Martin, Maurice (1980) 'Egypte, les modes informels du changement', Etudes, avril, pp. 435-52.

Mathieu, Lilian (2002) 'Rapport au politique, dimensions cognitives et perspectives pragmatiques dans l'analyse des mouvements sociaux', Revue française de science politique, 52 (1), pp. 75-100.

Tilly, Charles (1978) From Mobilization to Revolution, Reading (Mass.), Addison Wesley.

Sawicki, Frédéric (2003) 'Les temps de d'engagement. A propos de l'institutionnalisation d'une association de défense de l'environnement', in Lagroye, Jacques (dir.) La politisation, Paris, Belin, pp. 123-146.

Sevgat, Bedran (2001) Diyarbakir Zindani, Istanbul, Mem Yayınları.

TBMM (1995) Faili Meçhul Siyasal Cinayetleri Araftırma Komisyon Raporları, Ankara, Ayyıldız Yayınları.

T.C. Diyarbakır Devlet Güvenlik Mahkemesi Cumhuriyet Başsavclığı (2000) İddianame, Hazırlık 2000/143.

Türkiye'deki Anarşi ve Terörün Gelismesi, Sonuçlari ve Güvenlik Kuvvetler ile Önlenmesi (1982) Ankara, Başbakanlık Basımevi.

White, Paul (2000) Primitive Rebels of Revolutionary Modernizers? The Kurdish National Movement in Turkey, London, Zed Books.

Aydınlık, quotidien 1 mai 1993-1 mai 1994, hebdo mai 94-février 2000. numéro du 19 Ekim 1997 avec rapport du MIT.

Cumhuriyet

2000'e Doğru, 1987-1993

Hira, depuis 1991

Kurdistan Report, depuis 1982

Özgür Gündem

Radikal

Tevhid

The Ummah. An independant Monthly Muslim Critique, Ankara (1978- ?). 


\section{Biographies}

Abdullah Öcalan est un kurde de Hilvan, Siverek, dans la province d'Urfa. Né en 1949, il est originaire d'une famille pauvre d'agriculteurs assez religieux. II fait de la prison après le coup d'État de 1971 (sept mois) Etudiant à la faculté de sciences politiques d'Ankara, il forme en 1974 l'Association pour l'éducation supérieure d'Ankara (AYOD: Ankara Yüksek Öğretim Derneği). II s'impose comme leader d'un petit groupe d'étudiants qui formera le PKK en 1978. Avec un petit groupe de militant, il passe en Syrie quelques mois avant le coup d'État de 1980 et commence les opérations de guérilla sur le territoire turc à partir de 1984. En juin 1988, il déclare déjà aux autorités turques qu'il serait prêt à un cessez-le-feu en échange de l'ouverture de négociations. En 1993, 1995 et 1998, il proclame trois cessez-le-feu unilatéraux successifs qui dureront quelques mois. Forcé de quitter la Syrie en 1998 à la suite de pressions turques, Öcalan part pour Moscou, puis Rome. L'Italie refuse son extradition vers la Turquie, en raison de la non-abolition de la peine de mort dans ce pays. En janvier 1999, il tente sans succès de rejoindre les PaysBas. Öcalan est finalement kidnappé par les services turcs, grâce à l'appui des États-Unis, au moment où il quitte l'ambassade de Grèce au Kenya et est emprisonné en Turquie le 16 février 1999. Condamné à mort le 29 juin 1999, sa sentence n'est pas appliquée. Le 3 août, il demande au PKK de mettre fin à la lutte armée.

Hüseyin Velioğlu (Abi). Né en 1952 dans la province de Batman (village d'Hirro, ilçe de Gercüş), de la tribu Habizbin. Sa famille, religieuse et respectée, n'a pas de propriété foncière importante. Différents témoignages le décrivent comme une personnalité effacée. Un de ses oncles et un de ses frères deviendront muhtar (maire de quartier) à Batman dans les années 90. II suit l'école primaire à Devrim Illkokulu, puis le lycée à Batman. II est fait mention de lui uniquement en 1972 quand il est puni pour avoir fumé. Etudiant à l'Ankara Siyasal Bilgiler Fakültesi Maliye Bölümü, et co-disciple d'Öcalan, il obtient de bons résultats scolaires. II est membre de l'organisation Milli Türk Talebe Birligi et des jeunes du Milli Selamet Partisi, les Akıncilar. II est également connu dans les milieux ülkücü (Bulut Faraç 79). II s'impose dans les groupes de discussion à Batman notamment parce qu'il a plus d'expérience et d'éducation que ses camarades. En février 1978, il fonde une revue islamiste à Ankara dont il devient directeur, The Ummah. An 
Dorronsoro, Gilles ; Grojean, Olivier (2004) 'Engagement militant et phénomènes de radicalisation chez les Kurdes de Turquie', European Journal of Turkish Studies, URL: http://www.ejts.org/document198.html To quote a passage, use paragraph (§).

independant Monthly Muslim Critique. En 1979, il rate l'examen pour devenir kaymakam (sous-préfet) et revient à Batman. II se présente pour la première et la dernière fois à une élection le 17 mai 1980 pour la présidence de la branche locale de Petrol-Iş. La gauche gagne avec 1600 voix contre 1100. II part alors pour Diyarbakır, s'installe dans le quartier de Kaynartepe et participe au groupe qui se constitue autour de la librairie Menzil. II rencontre un Frère Musulman syrien, échappé du massacre de Hama de 1982 et réfugié à KIzltepe, Molla Ahmet, ce qui renforce sa proximité avec ce courant (DGM 2000/143/27). II ouvre la librairie lilim dans la province de Diyarbakır avec son compagnon İsa Altsoy. En 1986, il ouvre une nouvelle librairie à Diyarbakır (avec Yasin Ekinci, fils de Abdulvahap Ekinci). II est devenu un islamiste localement connu et construit un réseau islamiste à partir notamment de sa librairie llim kitabevi. II vit à Mardin pour l'essentiel jusqu'en 1996, puis part s'installer dans le quartier de Beykoz à Istanbul, où il trouvera la mort lors d'une opération de police en janvier 2000. 